\title{
General form for designing two-dimensional quadrantally symmetric linear-phase FIR digital filters by analytical least-squares method
}

\author{
Soo-Chang Pei ${ }^{\mathrm{a}, *}$, Jong-Jy Shyu ${ }^{\mathrm{b}}$ \\ ${ }^{a}$ Department of Electrical Engineering, National Taiwan University, Taipei, Taiwan, ROC \\ ${ }^{'}$ Department of Computer Science and Engineering, Tatung Institute of Technology, Taipei, Taiwan, ROC
}

Received 22 April 1994; revised 13 October 1994

\begin{abstract}
The analytical least-squares method has been generalized and extended for designing 16 types of two-dimensional FIR filters with quadrantally symmetric or antisymmetric frequency responses. By means of a closed-form transformation matrix, this fast design method can determine the filter's coefficients very effectively without a recourse to an iterative optimization technique or matrix inversion. Design examples are presented to illustrate the simplicity and efficiency of the proposed method.
\end{abstract}

\section{Zusammenfassung}

Die analytische Kleinste-Quadrat-Methode wird verallgemeinert und erweitert zum Entwurf von 16 Arten von zweidimensionalen FIR-Filtern mit quadrantenweise gerade oder ungerade symmetrischen Frequenzgängen. Mit Hilfe einer Transformationsmatrix in geschlossener Form kann man nach dieser Schnellen Entwurfsmethode die Filterkoeffizienten sehr effizient ohne Rückgriff auf eine iterative Optimierungstechnik oder eine Matrizeninversion bestimmen. Entwurfsbeispiele werden zur Erlaüterung der Einfachheit und Wirksamkeit des vorgeschlagenen Verfahrens vorgestellt.

\section{Résumé}

La méthode analytique par les moindres carrés a été généralisée et étendue pour la réalisation de 16 types de filtres FIR bidimensionnels à résponse fréquentielle symétrique ou antisymétrique. Par l'utilisation d'une matrice de transformation close, cette méthode de conception rapide peut déterminer les coefficients du filtre facilement sans avoir recours à une technique d'optimisation itérative ou à une inversion de matrice. Des exemples de réalisation sont présentés pour illustrer la simplicité et l'efficacité de la méthode proposée.

Keywords: Least-squares method; FIR filter

\footnotetext{
*Corresponding author. Tel.: + 886-2-3635251 Ext 321; fax: + 886-2-3638247; e-mail: pei@cc.ee.ntu.edu.tw.
} 


\section{Introduction}

Recently, there has been increasing interest in using the two-dimensional (2-D) digital filters in processing a variety of $2-D$ filtering for processing seismic records, gravity and magnetic data. Twodimensional filtering can also be used for the enhancement of photographic data such as weather photos, air photos and medical X-ray images. Twodimensional FIR filters are often used for all these applications, since it is easy to get the desired magnitude and linear phase responses in 2-D frequency domain, and also does not have the problem of stability to prevent overflow in computation.

Many techniques for designing 2-D linear-phase FIR filters have been reported in the literature [2-7]. Most of the design techniques employ some iterative optimization procedures or large matrix inversions to achieve the design solution. These algorithms suffer from heavy computation load and the slow convergence to the correct solutions. Recently, Ahmad and Wang [1] presented an analytical solution to the least-squares error design of 2-D FIR filters with quadrantally symmetric or antisymmetric frequency responses. By means of simple closed-form transformation matrix, this novel design technique allows the determination of filter's coefficients directly from its frequency response specification. The unique advantage of this technique is that it is very simple and fast without employing iterative optimization procedures and matrix inversion. However, Ahmad and Wang's method is limited to two special types of 2-D FIR filter designs [1]. This paper will show a general form for designing 2-D linear-phase FIR digital filters by analytical least-squares method, in which the general 16 types of 2-D filters can be easily designed, and the results are very satisfactory.

\section{Problem formulation}

The frequency response of a 2-D FIR filter with the impulse response $h\left(n_{1}, n_{2}\right), n_{1}=0,1,2, \ldots, \hat{N}_{1}-1$, $n_{2}=0,1,2, \ldots, \hat{N}_{2}-1$, can be characterized as

$$
\hat{H}\left(\omega_{1}, \omega_{2}\right)=\sum_{n_{1}=0}^{\hat{N}_{1}-1} \sum_{n_{2}=0}^{\hat{N}_{2}-1} h\left(n_{1}, n_{2}\right) \mathrm{e}^{-\mathrm{j} n_{1} \omega_{1}} \mathrm{e}^{-\mathrm{j} n_{2} \omega_{2}}
$$

If $h\left(n_{1}, n_{2}\right)$ satisfies certain symmetric condition [8], Eq. (1) can be rewritten as

$$
\begin{aligned}
\hat{H}\left(\omega_{1}, \omega_{2}\right)= & \mathrm{e}^{-\mathrm{j}\left[\left(\hat{N}_{1}-1\right) / 2\right] \omega_{1}} \mathrm{e}^{-\mathrm{j}\left[\left(\hat{N}_{2}-1\right) / 2\right] \omega_{2}} \mathrm{e}^{\mathrm{j}(L \pi) / 2} \\
& \times H\left(\omega_{1}, \omega_{2}\right)
\end{aligned}
$$

where

$L= \begin{cases}0 & \text { for Type I filters, } \\ 1 & \text { for Type II and III filters, } \\ 2 & \text { for Type IV filters, }\end{cases}$

and $H\left(\omega_{1}, \omega_{2}\right)$ is a real-valued function. Notice that by excluding the linear-phase part in Eq. (2), the frequency responses are real-valued functions for Type I even-even and Type IV odd-odd sequences, and are imaginary-valued functions for Type II even-odd and Type III odd-even sequences. For example, if $h\left(n_{1}, n_{2}\right)$ is a Type I even-even 2-D sequence, $\hat{N}_{1}$ and $\hat{N}_{2}$ are odd integers, then

$$
\begin{aligned}
& H\left(\omega_{1}, \omega_{2}\right) \\
& \quad=\sum_{n_{1}=0}^{\left(N_{1}-1\right) / 2} \sum_{n_{2}=0}^{\left(N_{2}-1\right) / 2} a\left(n_{1}, n_{2}\right) \cos \left(n_{1} \omega_{1}\right) \cos \left(n_{2} \omega_{2}\right),
\end{aligned}
$$

which is a real-valued function and $a\left(n_{1}, n_{2}\right)$ are related to $h\left(n_{1}, n_{2}\right)$ by

$$
\begin{aligned}
& a(0,0)=h\left(\frac{\hat{N}_{1}-1}{2}, \frac{\hat{N}_{2}-1}{2}\right), \\
& a\left(0, n_{2}\right)=2 h\left(\frac{\hat{N}_{1}-1}{2}, \frac{\hat{N}_{2}-1}{2}-n_{2}\right), \\
& n_{2}=1, \ldots, \frac{\hat{N}_{2}-1}{2}, \\
& a\left(n_{1}, 0\right)=2 h\left(\frac{\hat{N}_{2}-1}{2}-n_{1}, \frac{\hat{N}_{2}-1}{2}\right), \\
& \quad n_{1}=1, \ldots, \frac{\hat{N}_{1}-1}{2}, \\
& a\left(n_{1}, n_{2}\right)=4 h\left(\frac{\hat{N}_{1}-1}{2}-n_{1}, \frac{\hat{N}_{2}-1}{2}-n_{2}\right), \\
& \quad n_{1}=1, \ldots, \frac{\hat{N}_{1}-1}{2}, \\
& \quad n_{2}=1, \ldots, \frac{\hat{N}_{2}-1}{2} .
\end{aligned}
$$

Therefore, according to the four types of 2-D sequences and their even/odd lengths $\left(\hat{N}_{1} \times \hat{N}_{2}\right)$, there are 16 different kinds of $H\left(\omega_{1}, \omega_{2}\right)$ which are tabulated in Table 1. The relationships between the 
Table 1

$H\left(\omega_{1}, \omega_{2}\right)$ of 2-D sequence with length $\hat{N}_{1} \times \hat{N}_{2}\left(N_{i}=\frac{1}{2}\left(\hat{N}_{i}-1\right)\right)$ for odd $\hat{N}_{i}$ and $N_{i}=\frac{1}{2} \hat{N}_{i}$ for even $\left.\hat{N}_{i}, i=1,2\right)$

\begin{tabular}{|c|c|c|c|}
\hline Type & Subtype & $\hat{N}_{1}, \hat{N}_{2}$ & $H\left(\omega_{1}, \omega_{2}\right)$ \\
\hline I & $\begin{array}{l}1 \\
2 \\
3 \\
4\end{array}$ & $\begin{array}{l}\hat{N}_{1}: \text { odd }, \hat{N}_{2}: \text { odd } \\
\hat{N}_{1}: \text { odd, } \hat{N}_{2}: \text { even } \\
\hat{N}_{1}: \text { even, } \hat{N}_{2}: \text { odd } \\
\hat{N}_{1}: \text { even, } \hat{N}_{2}: \text { even }\end{array}$ & $\begin{array}{l}\sum_{n_{1}=0}^{N_{1}} \sum_{n_{2}=0}^{N_{2}} a\left(n_{1}, n_{2}\right) \cos \left(n_{1}\left(r_{1}\right) \cos \left(n_{2}\left(\omega_{2}\right)\right.\right. \\
\left.\sum_{n_{1}=0}^{N_{1}} \sum_{n_{2}=1}^{N_{2}} a\left(n_{1}, n_{2}\right) \cos \left(n_{1} \omega_{1}\right) \cos \left(n_{2}-\frac{1}{2}\right) \omega_{2}\right) \\
\sum_{n_{1}}^{N_{1}} \sum_{n_{1}}=1 \sum_{n_{2}=0}^{N_{2}} a\left(n_{1}, n_{2}\right) \cos \left(\left(n_{1}-\frac{1}{2}\right) \omega_{1}\right) \cos \left(n_{2} \omega_{2}\right) \\
\sum_{n_{1}=1}^{N_{1}} \sum_{n_{2}=1}^{N_{2}} a\left(n_{1}, n_{2}\right) \cos \left(\left(n_{1}-\frac{1}{2}\right) \omega_{1}\right) \cos \left(\left(n_{2}-\frac{1}{2}\right) \omega_{2}\right)\end{array}$ \\
\hline II & $\begin{array}{l}1 \\
2 \\
3 \\
4\end{array}$ & $\begin{array}{l}\hat{N}_{1}: \text { odd, } \hat{N}_{2}: \text { odd } \\
\hat{N}_{1}: \text { odd, } \hat{N}_{2}: \text { even } \\
\hat{N}_{1}: \text { even, } \hat{N}_{2}: \text { odd } \\
\hat{N}_{1}: \text { even, } \hat{N}_{2}: \text { even }\end{array}$ & $\begin{array}{l}\sum_{n_{1}=0}^{N_{1}} \sum_{n_{2}=1}^{N_{2}} a\left(n_{1}, n_{2}\right) \cos \left(n_{1} \omega_{1}\right) \sin \left(n_{2} \omega_{2}\right) \\
\sum_{n_{1}=0}^{N_{1}} \sum_{n_{2}=1}^{N_{2}} a\left(n_{1}, n_{2}\right) \cos \left(n_{1} \omega_{1}\right) \sin \left(\left(n_{2}-\frac{1}{2}\right) \omega_{2}\right) \\
\sum_{n_{1}=1}^{N_{1}} \sum_{n_{2}=1}^{N_{2}} a\left(n_{1}, n_{2}\right) \cos \left(\left(n_{1}-\frac{1}{2}\right) \omega_{1}\right) \sin \left(n_{2} \omega_{2}\right) \\
\sum_{n_{1}=1}^{N_{1}} \sum_{n_{2}=1}^{N_{2}} a\left(n_{1}, n_{2}\right) \cos \left(\left(n_{1}-\frac{1}{2}\right) \omega_{1}\right) \sin \left(\left(n_{2}-\frac{1}{2}\right) \omega_{2}\right)\end{array}$ \\
\hline III & $\begin{array}{l}1 \\
2 \\
3 \\
4\end{array}$ & $\begin{array}{l}\hat{N}_{1}: \text { odd }, \hat{N}_{2}: \text { odd } \\
\hat{N}_{1}: \text { odd }, \hat{N}_{2}: \text { even } \\
\hat{N}_{1}: \text { even, }, \hat{N}_{2}: \text { odd } \\
\hat{N}_{1}: \text { even, } \hat{N}_{2}: \text { even }\end{array}$ & $\begin{array}{l}\sum_{n_{1}=1}^{N_{1}} \sum_{n_{2}=0}^{N_{2}} a\left(n_{1}, n_{2}\right) \sin \left(n_{1} \omega_{1}\right) \cos \left(n_{2} \omega_{2}\right) \\
\sum_{n_{1}}^{N_{1}} \sum_{n_{2}}^{N_{2}} \sum_{2=1} a\left(n_{1}, n_{2}\right) \sin \left(n_{1} \omega_{1}\right) \cos \left(\left(n_{2}-\frac{1}{2}\right) \omega_{2}\right) \\
\sum_{n_{1}}^{N_{1}} \sum_{n_{1}}^{N_{2}} \sum_{n_{2}=0} a\left(n_{1}, n_{2}\right) \sin \left(\left(n_{1}-\frac{1}{2}\right) \omega_{1}\right) \cos \left(n_{2} \omega_{2}\right) \\
\sum_{n_{1}=1}^{N_{1}} \sum_{n_{2}=1}^{N_{2}} a\left(n_{1}, n_{2}\right) \sin \left(\left(n_{1}-\frac{1}{2}\right) \omega_{1}\right) \cos \left(\left(n_{2}-\frac{1}{2}\right) \omega_{2}\right)\end{array}$ \\
\hline IV & $\begin{array}{l}1 \\
2 \\
3 \\
4\end{array}$ & $\begin{array}{l}\hat{N}_{1}: \text { odd }, \hat{N}_{2}: \text { odd } \\
\hat{N}_{1}: \text { odd }, \hat{N}_{2}: \text { even } \\
\hat{N}_{1}: \text { even, } \hat{N}_{2}: \text { odd } \\
\hat{N}_{1}: \text { even, } \hat{N}_{2}: \text { even }\end{array}$ & $\begin{array}{l}\sum_{n_{1}=1}^{N_{1}} \sum_{n_{2}=1}^{N_{2}} a\left(n_{1}, n_{2}\right) \sin \left(n_{1} \omega_{1}\right) \sin \left(n_{2} \omega_{2}\right) \\
\sum_{n_{1}=1}^{N_{1}} \sum_{n_{2}=1}^{N_{2}} a\left(n_{1}, n_{2}\right) \sin \left(n_{1} \omega_{1}\right) \sin \left(\left(n_{2}-\frac{1}{2}\right) \omega_{2}\right) \\
\sum_{n_{1}=1}^{N_{1}} \sum_{n_{2}=1}^{N_{2}} a\left(n_{1}, n_{2}\right) \sin \left(\left(n_{1}-\frac{1}{2}\right) \omega_{1}\right) \sin \left(n_{2} \omega_{2}\right) \\
\sum_{n_{1}=1}^{N_{1}=1} \sum_{n_{2}=1}^{N} a\left(n_{1}, n_{2}\right) \sin \left(\left(n_{1}-\frac{1}{2}\right) \omega_{1}\right) \sin \left(\left(n_{2}-\frac{1}{2}\right) \omega_{2}\right)\end{array}$ \\
\hline
\end{tabular}

coefficients $a\left(n_{1}, n_{2}\right)$ in $H\left(\omega_{1}, \omega_{2}\right)$ and $h\left(n_{1}, n_{2}\right)$ are listed in Table 2. Although the four types of even/odd, symmetric/antisymmetric 1-D linearphase FIR filters are well known [9], the above 16 types of 2-D FIR filters have not been well studied and exploited in the open literature [8]. For example, Ahmad and Wang have only discussed the even-even quadrantally symmetric and oddodd antisymmetric types of 2-D FIR filter designs [1]. In this paper, we will extend the analytic leastsquares method to design the above general 16 types of 2-D FIR filters.

Let the square error sum between the specified desired frequency response $H_{\mathrm{d}}(i \pi / M, j \pi / M)$ and the actual filter response $H(i \pi / M, j \pi / M)$ be defined as

$$
\begin{aligned}
E & =\sum_{i=0}^{M} \sum_{j=0}^{M}\left[H_{\mathrm{d}}\left(\frac{i \pi}{M}, \frac{j \pi}{M}\right)-H\left(\frac{i \pi}{M}, \frac{j \pi}{M}\right)\right]^{2} \\
& =\operatorname{tr}\left[\left(\boldsymbol{H}_{\mathrm{d}}-\boldsymbol{H}^{\mathrm{T}}\left(\boldsymbol{H}_{\mathrm{d}}-\boldsymbol{H}\right)\right]\right. \\
& =\operatorname{tr}\left[\boldsymbol{H}_{\mathrm{d}}^{\mathrm{T}} \boldsymbol{H}_{\mathrm{d}}-2 \boldsymbol{H}_{\mathrm{d}}^{\mathrm{T}} \boldsymbol{H}+\boldsymbol{H}^{\mathrm{T}} \boldsymbol{H}\right],
\end{aligned}
$$

where $T$ denotes the transpose operation, $\boldsymbol{H}_{\mathrm{d}}=\left[H_{\mathrm{d}_{i j}}\right]$ and $\boldsymbol{H}=\left[H_{i j}\right],(i, j=0, \ldots, M)$ are $(M+1) \times(M+1)$ matrices whose elements are given, respectively, by

$H_{\mathrm{d}_{i j}}=H_{\mathrm{d}}\left(\frac{i \pi}{M}, \frac{j \pi}{M}\right)$

and

$H_{i j}=H\left(\frac{i \pi}{M}, \frac{j \pi}{M}\right)$.

Here an $(M+1) \times(M+1)$ rectangular grid is chosen for the evaluation of the amplitude response in the first quadrant of the $\left(\omega_{1}, \omega_{2}\right)$ plane. Generally, the matrix $\boldsymbol{H}$ can be represented as

$\boldsymbol{H}=\boldsymbol{P} A \boldsymbol{Q}^{\mathrm{T}}$,

where the matrix $A=\left[a_{i j}\right]$ specifies the filter's coefficients, whose dimensions and element ranges are listed in Table 3. The frequency response transformation matrices $\boldsymbol{P}$ and $\boldsymbol{Q}$ depend on the 
Table 2

Relationships between $a\left(n_{1}, n_{2}\right)$ in $H\left(\omega_{1}, \omega_{2}\right)$ and $h\left(n_{1}, n_{2}\right)$ in $H\left(\omega_{1}, \omega_{2}\right)$

\begin{tabular}{|c|c|}
\hline Type & Relationship between $a\left(n_{1}, n_{2}\right)$ and $h\left(n_{1}, n_{2}\right)$ \\
\hline I-1 & $\begin{array}{l}a(0,0)=h\left(N_{1}, N_{2}\right) \\
a\left(0, n_{2}\right)=2 h\left(N_{1}, N_{2}-n_{2}\right), \quad n_{2}=1, \ldots, N_{2} \\
a\left(n_{1}, 0\right)=2 h\left(N_{1}-n_{1}, N_{2}\right), \quad n_{1}=1, \ldots, N_{1} \\
a\left(n_{1}, n_{2}\right)=4 h\left(N_{1}-n_{1}, N_{2}-n_{2}\right), \quad n_{1}=1, \ldots, N_{1}, n_{2}=1, \ldots, N_{2}\end{array}$ \\
\hline I-2 & $\begin{array}{l}a\left(0, n_{2}\right)=2 h\left(N_{1}, N_{2}-n_{2}\right), \quad n_{2}=1, \ldots, N_{2} \\
a\left(n_{1}, n_{2}\right)=4 h\left(N_{1}-n_{1}, N_{2}-n_{2}\right), \quad n_{1}=1, \ldots, N_{1}, n_{2}=1, \ldots, N_{2}\end{array}$ \\
\hline I-3 & $\begin{array}{l}a\left(n_{1}, 0\right)=2 h\left(N_{1}-n_{1}, N_{2}\right), \quad n_{1}=1, \ldots, N_{1} \\
a\left(n_{1}, n_{2}\right)=4 h\left(N_{1}-n_{1}, N_{2}-n_{2}\right), \quad n_{1}=1, \ldots, N_{1}, n_{2}=1, \ldots, N_{2}\end{array}$ \\
\hline $\mathbf{I}-4$ & $a\left(n_{1}, n_{2}\right)=4 h\left(N_{1}-n_{1}, N_{2}-n_{2}\right), \quad n_{1}=1, \ldots, N_{1}, n_{2}=1, \ldots, N_{2}$ \\
\hline III-1 & $\begin{array}{l}h\left(n_{1}, N_{2}\right)=0, \quad n_{1}=0, \ldots, \hat{N}_{1}-1 \\
a\left(0, n_{2}\right)=2 h\left(N_{1}, N_{2}-n_{2}\right), \quad n_{2}-1, \ldots, N_{2} \\
a\left(n_{1}, n_{2}\right)=4 h\left(N_{1}-n_{1}, N_{2}-n_{2}\right), \quad n_{1}=1, \ldots, N_{1}, n_{2}=1, \ldots, N_{2}\end{array}$ \\
\hline II-2 & $\begin{array}{l}a\left(0, n_{2}\right)=2 h\left(N_{1}, N_{2}-n_{2}\right), \quad n_{2}=1, \ldots, N_{2} \\
a\left(n_{1}, n_{2}\right)=4 h\left(N_{1}-n_{1}, N_{2}-n_{2}\right), \quad n_{1}=1, \ldots, N_{1}, n_{2}=1, \ldots, N_{2}\end{array}$ \\
\hline II-3 & $\begin{array}{l}h\left(n_{1}, N_{2}\right)=0, \quad n_{1}=0, \ldots, \widehat{N}_{1}-1 \\
a\left(n_{1}, n_{2}\right)=4 h\left(N_{1}-n_{1}, N_{2}-n_{2}\right), \quad n_{1}=1, \ldots, N_{1}, n_{2}=1, \ldots, N_{2}\end{array}$ \\
\hline II-4 & $a\left(n_{1}, n_{2}\right)=4 h\left(N_{1}-n_{1}, N_{2}-n_{2}\right), \quad n_{1}=1, \ldots, N_{1}, n_{2}=1, \ldots, N_{2}$ \\
\hline III-1 & $\begin{array}{l}h\left(N_{1}, n_{2}\right)=0, \quad n_{2}=0, \ldots, \hat{N}_{2}-1 \\
a\left(n_{1}, 0\right)=2 h\left(N_{1}-n_{1}, N_{2}\right), \quad n_{1}=1, \ldots, N_{1} \\
a\left(n_{1}, n_{2}\right)=4 h\left(N_{1}-n_{1}, N_{2}-n_{2}\right), \quad n_{1}=1, \ldots, N_{1}, n_{2}=1, \ldots, N_{2}\end{array}$ \\
\hline III-2 & $\begin{array}{l}h\left(N_{1}, n_{2}\right)=0, \quad n_{2}=0, \ldots, \hat{N}_{2}-1 \\
a\left(n_{1}, n_{2}\right)=4 h\left(N_{1}-n_{1}, N_{2}-n_{2}\right), \quad n_{1}=1, \ldots, N_{1}, n_{2}=1, \ldots, N_{2}\end{array}$ \\
\hline III-3 & $\begin{array}{l}a\left(n_{1}, 0\right)=2 h\left(N_{1}-n_{1}, N_{2}\right), \quad n_{1}=1, \ldots, N_{1} \\
a\left(n_{1}, n_{2}\right)=4 h\left(N_{1}-n_{1}, N_{2}-n_{2}\right), \quad n_{1}=1, \ldots, N_{1}, n_{2}=1, \ldots, N_{2}\end{array}$ \\
\hline III-4 & $a\left(n_{1}, n_{2}\right)=4 h\left(N_{1}-n_{1}, N_{2}-n_{2}\right), \quad n_{1}=1, \ldots, N_{1}, n_{2}=1, \ldots, N_{2}$ \\
\hline IV-1 & $\begin{array}{l}h\left(N_{1}, n_{2}\right)=0, \quad n_{2}=0, \ldots, \hat{N_{2}}-1 \\
h\left(n_{1}, N_{2}\right)=0, \quad n_{1}=0, \ldots, \hat{N}_{1}-1 \\
a\left(n_{1}, n_{2}\right)=4 h\left(N_{1}-n_{1}, N_{2}-n_{2}\right), \quad n_{1}=1, \ldots, N_{1}, n_{2}=1, \ldots, N_{2}\end{array}$ \\
\hline IV-2 & $\begin{array}{l}h\left(N_{1}, n_{2}\right)=0, \quad n_{2}=0, \ldots, \hat{N}_{2}-1 \\
a\left(n_{1}, n_{2}\right)=4 h\left(N_{1}-n_{1}, N_{2}-n_{2}\right), \quad n_{1}=1, \ldots, N_{1}, n_{2}=1, \ldots, N_{2}\end{array}$ \\
\hline IV-3 & $\begin{array}{l}h\left(n_{1}, N_{2}\right)=0, \quad n_{1}=0, \ldots, \hat{N}_{1}-1 \\
a\left(n_{1}, n_{2}\right)=4 h\left(N_{1}-n_{1}, N_{2} \quad n_{2}\right), \quad n_{1}=1, \ldots, N_{1}, n_{2}=1, \ldots, N_{2}\end{array}$ \\
\hline IV-4 & $a\left(n_{1}, n_{2}\right)=4 h\left(N_{1}-n_{1}, N_{2}-n_{2}\right), \quad n_{1}=1, \ldots, N_{1}, n_{2}=1, \ldots, N_{2}$ \\
\hline
\end{tabular}


Table 3

Dimensions and element ranges of the matrix $\boldsymbol{A}$ and corresponding tables for $\boldsymbol{S}$ and $\boldsymbol{V}$

\begin{tabular}{|c|c|c|c|c|c|}
\hline Type & Subtype & Dimension of $A$ & $\begin{array}{l}\text { Elcment range } \\
\text { of } \boldsymbol{A}\end{array}$ & $\begin{array}{l}\text { Corresponding } \\
\text { table for } S\end{array}$ & $\begin{array}{l}\text { Corresponding } \\
\text { table for } V\end{array}$ \\
\hline \multirow[t]{4}{*}{ I } & 1 & $\left(N_{1}+1\right) \times\left(N_{2}+1\right)$ & $\begin{array}{l}i=0,1, \ldots, N_{1} \\
j=0,1, \ldots, N_{2}\end{array}$ & IV & IV \\
\hline & 2 & $\left(N_{1}+1\right) \times N_{2}$ & $\begin{array}{l}i=0,1, \ldots, N_{1} \\
j=1,2, \ldots, N_{2}\end{array}$ & IV & $\mathrm{V}$ \\
\hline & 3 & $N_{1} \times\left(N_{2}+1\right)$ & $\begin{array}{l}i=1,2, \ldots, N_{1} \\
j=0,1, \ldots, N_{2}\end{array}$ & $\mathrm{~V}$ & IV \\
\hline & 4 & $N_{1} \times N_{2}$ & $\begin{array}{l}i=1,2, \ldots, N_{1} \\
j=1,2, \ldots, N_{2}\end{array}$ & V & V \\
\hline \multirow[t]{4}{*}{ II } & 1 & $\left(N_{1}+1\right) \times N_{2}$ & $\begin{array}{l}i=0,1, \ldots, N_{1} \\
j=1,2, \ldots, N_{2}\end{array}$ & IV & VI \\
\hline & 2 & $\left(N_{1}+1\right) \times N_{2}$ & $\begin{array}{l}i=0,1, \ldots, N_{1} \\
j=1,2, \ldots, N_{2}\end{array}$ & IV & VII \\
\hline & 3 & $N_{1} \times N_{2}$ & $\begin{array}{l}i=1,2, \ldots, N_{1} \\
j=1,2, \ldots, N_{2}\end{array}$ & V & VI \\
\hline & 4 & $N_{1} \times N_{2}$ & $\begin{array}{l}i=1,2, \ldots, N_{1} \\
j=1,2, \ldots, N_{2}\end{array}$ & V & VII \\
\hline \multirow[t]{4}{*}{ III } & 1 & $N_{1} \times\left(N_{2}+1\right)$ & $\begin{array}{l}i=1,2, \ldots, N_{1} \\
j=0,1, \ldots, N_{2}\end{array}$ & VI & IV \\
\hline & 2 & $N_{1} \times N_{2}$ & $\begin{array}{l}i=1,2, \ldots, N_{1} \\
j=1,2, \ldots, N_{2}\end{array}$ & VI & $\mathrm{V}$ \\
\hline & 3 & $N_{1} \times\left(N_{2}+1\right)$ & $\begin{array}{l}i=1,2, \ldots, N_{1} \\
j=0,1, \ldots, N_{2}\end{array}$ & VII & IV \\
\hline & 4 & $N_{1} \times N_{2}$ & $\begin{array}{l}i=1,2, \ldots, N_{1} \\
j=1,2, \ldots, N_{2}\end{array}$ & VII & V \\
\hline \multirow[t]{4}{*}{ IV } & 1 & $N_{1} \times N_{2}$ & $\begin{array}{l}i=1,2, \ldots, N_{1} \\
j=1,2, \ldots, N_{2}\end{array}$ & VI & VI \\
\hline & 2 & $N_{1} \times N_{2}$ & $\begin{array}{l}i=1,2, \ldots, N_{1} \\
j=1,2, \ldots, N_{2}\end{array}$ & VI & VII \\
\hline & 3 & $N_{1} \times N_{2}$ & $\begin{array}{l}i=1,2, \ldots, N_{1} \\
j=1,2, \ldots, N_{2}\end{array}$ & VII & VI \\
\hline & 4 & $N_{1} \times N_{2}$ & $\begin{array}{l}i=1,2, \ldots, N_{1} \\
j=1,2, \ldots, N_{2}\end{array}$ & VII & VII \\
\hline
\end{tabular}

two separable functions in $\omega_{1}$ and $\omega_{2}$, respectively

For example, for Type I-1 digital filter design,

$\boldsymbol{P}=\left[\cos \left(\frac{i j \pi}{M}\right), i=0,1, \ldots, M, j=0,1, \ldots, N_{1}\right]$,

and

$\boldsymbol{Q}=\left[\cos \left(\frac{i j \pi}{M}\right), i=0,1, \ldots, M, j=0,1, \ldots, N_{2}\right]$, but for Type II-4 filter design,

$$
\begin{aligned}
& \boldsymbol{P}=\left[\cos \left(\frac{i\left(j-\frac{1}{2}\right) \pi}{M}\right),\right. \\
& \text { and } \\
& \left.\qquad i=0,1, \ldots, M, j=1,2, \ldots, N_{1}\right]
\end{aligned}
$$

$$
\begin{aligned}
\boldsymbol{Q}=\left[\sin \left(\frac{i\left(j-\frac{1}{2}\right) \pi}{M}\right)\right. \\
\\
\left.i=0,1, \ldots, M, j=1,2, \ldots, N_{2}\right]
\end{aligned}
$$


where $N_{1}$ and $N_{2}$ are defined in Table 1 . Substitution of (9) into (6) yields

$E=\operatorname{tr}\left[H_{\mathrm{d}}^{\mathrm{T}} \boldsymbol{H}_{\mathrm{d}}-2 \boldsymbol{H}_{\mathrm{d}}^{\mathrm{T}} \boldsymbol{P A} \boldsymbol{Q}^{\mathrm{T}}+\left(\boldsymbol{P} \boldsymbol{A} \boldsymbol{Q}^{\mathrm{T}}\right)^{\mathrm{T}} \boldsymbol{P} \boldsymbol{A} \boldsymbol{Q}^{\mathrm{T}}\right]$.

when $\partial E / \partial A=0$, the minimum error is obtained and the closed-form solution for $A$ is given by

$\boldsymbol{A}=\left(\boldsymbol{P}^{\mathrm{T}} \boldsymbol{P}\right)^{-1} \boldsymbol{P}^{\mathrm{T}} \boldsymbol{H}_{\mathrm{d}}\left(\left(\boldsymbol{Q}^{\mathrm{T}} \boldsymbol{Q}\right)^{-1} \boldsymbol{Q}^{\mathrm{T}}\right)^{\mathrm{T}}$

Let $\boldsymbol{R}=\boldsymbol{P}^{\mathrm{T}} \boldsymbol{P}$ and $\boldsymbol{U}=\boldsymbol{Q}^{\mathrm{T}} \boldsymbol{Q}$, then

$A=R^{-1} P^{\mathrm{T}} H_{\mathrm{d}}\left(U^{-1} Q^{\mathrm{T}}\right)^{\mathrm{T}}$.

Moreover, let $S=R^{-1} P^{\mathrm{T}}$ and $V=U^{-1} Q^{\mathrm{T}}(S$ and $V$ are called the inverse frequency response transformation matrices), then

$\boldsymbol{A}=\boldsymbol{S} \boldsymbol{H}_{\mathrm{d}} \boldsymbol{V}^{\mathrm{T}}$.

In Appendix A and [8], it will be shown that as a consequence of the symmetry and matrix properties, the number of operations in the computation of $\boldsymbol{S}$ and $\boldsymbol{V}$ is significantly reduced. Clearly, there are four cases for finding the matrix elements

Table 4

Elements of the inverse frequency response transformation matrix when the elements in the corresponding $\boldsymbol{P}$ or $\boldsymbol{Q}$ are $\cos (i l \pi / M)\left(0 \leqslant i \leqslant M, 0 \leqslant l \leqslant N_{k}\right)$

\begin{tabular}{lll}
\hline$i\left(0 \leqslant i \leqslant N_{k}\right)$ & $l(0 \leqslant l \leqslant M)$ & $T_{i l}$ \\
\hline 0 & $0, M$ & $f_{1}$ \\
0 & $0<l<M$ & {$\left[f_{1}+f_{2}(i, l)-2 f_{1} f_{3}(l)\right] / M$} \\
even & $0, M$ & $2 f_{2}(i, l)$ \\
even & $0<l<M$ & $2\left[f_{1}+f_{2}(i, l)-2 f_{1} f_{3}(l)\right] / M$ \\
odd & 0 & $f_{4}$ \\
odd & $M$ & $-f_{4}$ \\
odd & $0<l<M$ & $2\left\{f_{2}(i, l)+f_{4}\left[f_{3}(l)-f_{5}(l)\right]\right\} / M$ \\
\hline
\end{tabular}

Table 5

Elements of the inverse frequency response transformation matrix when the elements in the corresponding $\boldsymbol{P}$ or $\boldsymbol{Q}$ are $\cos \left(i\left(l-\frac{1}{2}\right) \pi / M\right)\left(0 \leqslant i \leqslant M, 1 \leqslant l \leqslant N_{k}\right)$

\begin{tabular}{lll}
\hline$i\left(1 \leqslant i \leqslant N_{k}\right)$ & $l(0 \leqslant l \leqslant M)$ & $T_{i l}$ \\
\hline $1 \leqslant i \leqslant N_{k}$ & 0 & $\frac{2}{M+N_{k}}$ \\
$1 \leqslant i \leqslant N_{k}$ & $M$ & 0 \\
$1 \leqslant i \leqslant N_{k}$ & $0<l<M$ & $\frac{2 f_{2}\left(i-\frac{1}{2}, l\right)}{M}-\frac{f_{6}\left(N_{k}, l\right)}{M\left(M+N_{k}\right) f_{6}\left(\frac{1}{2}, l\right)}$ \\
\hline
\end{tabular}

of $S$ and $V$, in which two of them involving $\cos (i j \pi / M)$ and $\sin (i j \pi / M)$ elements have been obtained in [1], and the one with $\cos \left(i\left(j-\frac{1}{2}\right) \pi / M\right)$ element is simplified in Appendix $A$, and the one with $\sin \left(i\left(j-\frac{1}{2}\right) \pi / M\right)$ element can be derived in the same manner. The summary results are listed in Tables 4-7, and for each of the 16 types of 2-D FIR filter designs, the corresponding tables for both $\boldsymbol{S}$ and $\boldsymbol{V}$ are tabulated in Table 3. Notice that the functions $f_{1}, f_{2}(i, j), f_{3}(j), f_{4}, f_{5}(j)$ and $f_{6}(i, j)$, uscd to express the elements of the matrix $S$ or $V$ in Tables 4-7, can be obtained from Table 8 , and the elements of different kinds of matrices $S$ and $V$ are denoted by $T_{i j}$ in general. Moreover, $M>N_{1}, N_{2}$ is required.

Once the elements of $\boldsymbol{S}$ and $\boldsymbol{V}$ have been evaluated using Tables 4-7, (17) can be used to compute the filter coefficient matrix $A$, and the 2-D FIR filter design is completed.

Table 6

Elements of the inverse frequency response transformation matrix when the elements in the corresponding $\boldsymbol{P}$ or $\boldsymbol{Q}$ are $\sin (i l \pi / M)\left(0 \leqslant i \leqslant M, 1 \leqslant l \leqslant N_{k}\right)$

\begin{tabular}{lll}
\hline$i\left(1 \leqslant i \leqslant N_{k}\right)$ & $l(0 \leqslant l \leqslant M)$ & $T_{i t}$ \\
\hline $1 \leqslant i \leqslant N_{k}$ & $0, M$ & 0 \\
$1 \leqslant i \leqslant N_{k}$ & $0<l<M$ & $\frac{2 f_{6}(i, l)}{M}$ \\
\hline
\end{tabular}

Table 7

Elements of the inverse frequency response transformation matrix when the elements in the corresponding $\boldsymbol{P}$ or $\boldsymbol{Q}$ are $\sin \left(i\left(l-\frac{1}{2}\right) \pi / M\right)\left(0 \leqslant i \leqslant M, 1 \leqslant l \leqslant N_{k}\right)$

\begin{tabular}{lll}
\hline$i\left(1 \leqslant i \leqslant N_{k}\right)$ & $l(0 \leqslant l \leqslant M)$ & $T_{i l}$ \\
\hline $1 \leqslant i \leqslant N_{k}$ & 0 & 0 \\
even & $M$ & $\frac{-2}{M+N_{k}}$ \\
odd & $M$ & $\frac{2}{M+N_{k}}$ \\
even & $0<l<M$ & $\frac{2 f_{6}\left(i-\frac{1}{2}, l\right)}{M}+\frac{(-1)^{N_{k}+1} f_{6}\left(N_{k}, l\right)}{M\left(M+N_{k}\right) f_{2}\left(\frac{1}{2}, l\right)}$ \\
odd & $0<l<M$ & $\frac{2 f_{6}\left(i-\frac{1}{2}, l\right)}{M}+\frac{(-1)^{N_{k}} f_{6}\left(N_{k}, l\right)}{M\left(M+N_{k}\right) f_{2}\left(\frac{1}{2}, l\right)}$ \\
&
\end{tabular}


Table 8

Expressions for the functions used in Tables 4-7

\begin{tabular}{lll}
\hline Function & Even $N_{k}$ & Odd $N_{k}$ \\
\hline$f_{1}$ & $\frac{1}{M+N_{k}+1}$ & $\frac{1}{M+N_{k}}$ \\
$f_{2}(i, l)$ & $\cos (i l \pi / M)$ & $\cos (i l \pi / M)$ \\
$f_{3}(l)$ & $\frac{\cos \left(N_{k} l \pi / 2 M\right) \sin \left(\left(N_{k}+2\right) l \pi / 2 M\right)}{\sin (l \pi / M)}$ & $\frac{\cos \left(\left(N_{k}-1\right) l \pi / 2 M\right) \sin \left(\left(N_{k}+1\right) l \pi / 2 M\right)}{\sin (l \pi / M)}$ \\
$f_{4}$ & $\frac{2}{M+N_{k}}$ & $\frac{2}{M+N_{k}+1}$ \\
$f_{5}(l)$ & $\frac{\cos \left(N_{k} l \pi / 2 M\right) \sin \left(\left(N_{k}+1\right) l \pi / 2 M\right)}{\sin (l \pi / 2 M)}$ & $\frac{\cos \left(N_{k} l \pi / 2 M\right) \sin \left(\left(N_{k}+1\right) l \pi / 2 M\right)}{\sin (l \pi / 2 M)}$ \\
$f_{6}(i, l)$ & $\sin (i l \pi / M)$ & $\sin (i l \pi / M)$ \\
\hline
\end{tabular}

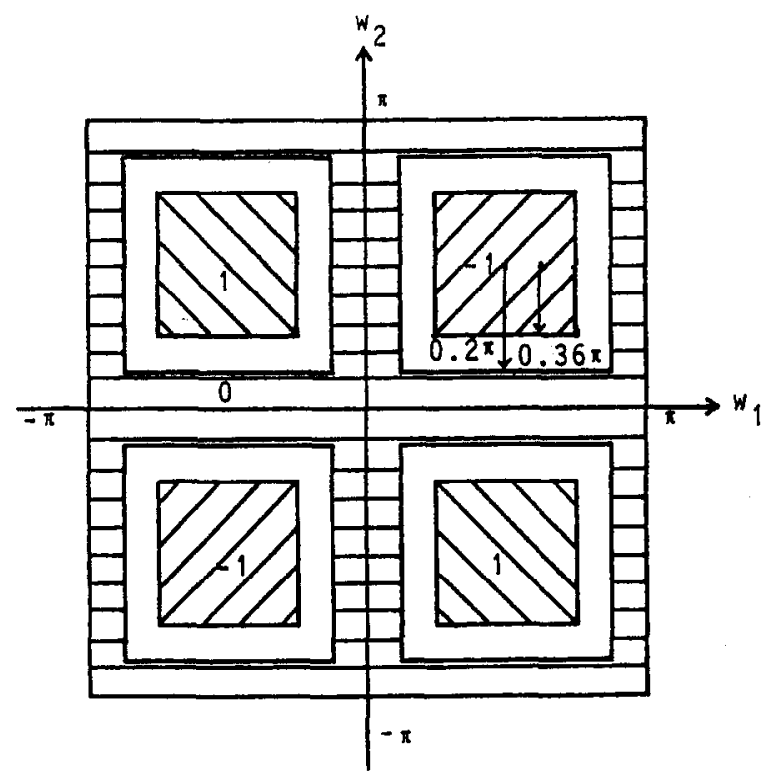

Fig. 1. Desired magnitude response specifications for designing a $21 \times 202-D$ Type IV-2 digital filter.

\section{Design example}

In this section, Type IV-2 2-D FIR filter is designed to demonstrate the effectiveness of this method. The desired filter specifications are shown

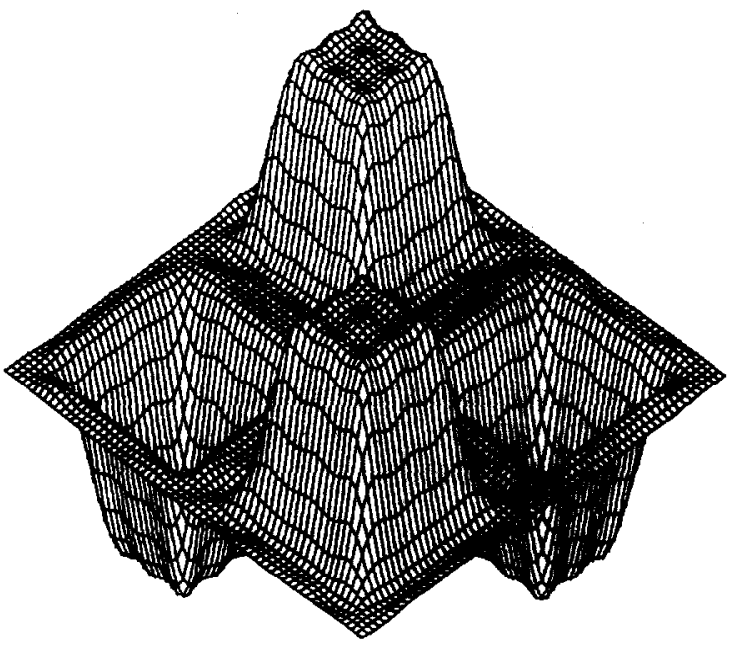

Fig. 2. The magnitude response of the designed $21 \times 202-\mathrm{D}$ Type IV-2 digital filter.

in Fig. 1, in which the regions with horizontal cross lines are the stopbands with desired response 0 , and those with north-east diagonal line are positive passband with desired response 1 , and those with north-west directional lines are negative passband with desired response -1 , and the others are the transition bands in which the magnitude varies linearly between 1 and 0 or -1 and 0 , respectively. When $N_{1}=N_{2}=10$ and $M=50$ are used, Fig. 2 
shows the resultant magnitude response, and the design only took about $0.18 \mathrm{~s}$ on VAX 8700 , which are much faster than the other optimization techniques.

\section{Conclusions}

A general form for designing 2-D FIR filters with quadrantally symmetric frequency response is presented in this paper. This analytical least squares method entails a number of closed-form transform matrices to simplify the filter design procedures greatly. Due to the simplicity of evaluating the functions in Table 8, another significant advantage of this design technique is that the design time increases very slowly as the filter order increases [1]. The designed 2-D filter's response is very satisfactory as illustrated through the presented numerical example.

Appendix A. Derivation of inverse frequency response transformation matrix $S$ or $V$ when the elements in $\boldsymbol{P}$ or $\boldsymbol{Q}$ are $\cos \left(i\left(l-\frac{1}{2}\right) \pi / M\right)$, $i=0,1, \ldots, M, l=1,2, \ldots, N_{k}(k=1$ or 2$)$.

In this section, matrix notation $\boldsymbol{Y}$ is used to represent $\boldsymbol{P}$ or $\boldsymbol{Q}$, and $N$ is used instead of $N_{1}$ or $N_{2}$. Hence $\boldsymbol{Y}=\left[Y_{i l}\right]$, where

$$
\begin{aligned}
Y_{i l} & =\cos \left(\frac{i\left(l-\frac{1}{2}\right) \pi}{M}\right), \\
i & =0,1, \ldots, M, \quad l=1,2, \ldots, N .
\end{aligned}
$$

Let

$$
\boldsymbol{Z}=\boldsymbol{Y}^{\mathrm{T}} \boldsymbol{Y}=\left[Z_{i l}, 1 \leqslant i, l \leqslant N\right],
$$

where

$$
\begin{aligned}
Z_{i l} & =\sum_{k=0}^{M} \cos \left(\frac{k\left(i-\frac{1}{2}\right) \pi}{M}\right) \cos \left(\frac{k\left(l-\frac{1}{2}\right) \pi}{M}\right) \\
& =\sum_{k=0}^{M-1} \cos \left(\frac{k\left(i-\frac{1}{2}\right) \pi}{M}\right) \cos \left(\frac{k\left(l-\frac{1}{2}\right) \pi}{M}\right), \\
i, l & =1,2, \ldots, N .
\end{aligned}
$$

Depending on the location of $Z_{i t}$ in the matrix, the derivation to obtain simplified expressions is divided into three cases.

Case 1. $1 \leqslant i=l \leqslant N$. Eq. (A.3) becomes

$$
\begin{aligned}
Z_{i l} & =\sum_{k=0}^{M-1} \cos ^{2}\left(\frac{k\left(i-\frac{1}{2}\right) \pi}{M}\right) \\
& =\frac{M}{2}+\frac{1}{2} \sum_{k=0}^{M-1} \cos \left(\frac{2 k\left(i-\frac{1}{2}\right) \pi}{M}\right) .
\end{aligned}
$$

By Eq. (A.1) of [1],

$\sum_{k=0}^{M-1} \cos \left(\frac{2 k\left(i-\frac{1}{2}\right) \pi}{M}\right)=1$,

so

$Z_{i l}=\frac{M+1}{2}$.

Case 2. $i \neq l$ and $(i+l)$ is even $((i-l)$ is even too $)$. Eq. (A.3) becomes

$$
\begin{aligned}
Z_{i l}=\frac{1}{2}\left[\sum_{k=0}^{M-1} \cos \left(\frac{k(i+l-1) \pi}{M}\right)\right. \\
\left.+\sum_{k=0}^{M-1} \cos \left(\frac{k(i-l) \pi}{M}\right)\right] .
\end{aligned}
$$

Similarly, by Eq. (A.1) of [1],

$$
\begin{aligned}
& \sum_{k=0}^{M-1} \cos \left(\frac{k(i+l-1) \pi}{M}\right)=1, \\
& \text { for } i+l \text { even, }
\end{aligned}
$$

and

$\sum_{k=0}^{M-1} \cos \left(\frac{k(i-l) \pi}{M}\right)=0$, for $i-l$ even,

so

$Z_{i l}=\frac{1}{2}$

Case 3. $i \neq l$ and $(i+l)$ is odd. Again, $Z_{i l}$ can be expressed as in (A.7), but it can be shown that

$\sum_{k=0}^{M-1} \cos \left(\frac{k(i+l-1) \pi}{M}\right)=0$, for $i+l$ odd (A.11) 
and

$\sum_{k=0}^{M-1} \cos \left(\frac{k(i-l) \pi}{M}\right)=1$, for $i-l$ odd

so

$Z_{i t}=\frac{1}{2}$

Hence,

$\boldsymbol{Z}=\left[\begin{array}{ccccc}\frac{1}{2}(M+1) & \frac{1}{2} & \frac{1}{2} & \cdots & \frac{1}{2} \\ \frac{1}{2} & \frac{1}{2}(M+1) & \frac{1}{2} & \cdots & \frac{1}{2} \\ \frac{1}{2} & \frac{1}{2} & \frac{1}{2}(M+1) & \cdots & \frac{1}{2} \\ \vdots & \vdots & \vdots & \ddots & \vdots \\ \frac{1}{2} & \frac{1}{2} & \frac{1}{2} & \cdots & \frac{1}{2}(M+1)\end{array}\right]=\frac{1}{2}\left[C+E E^{\mathrm{T}}\right]$

where $\boldsymbol{C}$ is an $N \times N$ matrix represented by

$\boldsymbol{C}=\left[\begin{array}{ccccc}M & 0 & 0 & \cdots & 0 \\ 0 & M & 0 & \cdots & 0 \\ 0 & 0 & M & \cdots & 0 \\ \vdots & \vdots & \vdots & \ddots & \vdots \\ 0 & 0 & 0 & \cdots & M\end{array}\right]$

and $\boldsymbol{E}$ is an $N \times 1$ matrix given by

$\boldsymbol{E}=\left[\begin{array}{lllll}1 & 1 & 1 & \ldots & 1\end{array}\right]^{\mathrm{T}}$

By Eq. (31) of [1],

$$
\begin{aligned}
Z^{-1} & =2 C^{-1}-2 \boldsymbol{C}^{-1} \boldsymbol{E}\left(1+\boldsymbol{E}^{\mathrm{T}} \boldsymbol{C}^{-1} \boldsymbol{E}\right)^{-1} \boldsymbol{E}^{\mathrm{T}} \boldsymbol{C}^{-1} \\
& =\left[\lambda_{i l}, i, l=1,2, \ldots, N\right]
\end{aligned}
$$

where

$$
\lambda_{i l}= \begin{cases}\frac{2(M+N-1)}{M(M+N)}, & i=l, \\ \frac{-2}{M(M+N)}, & i \neq l .\end{cases}
$$

Let $\boldsymbol{T}=\boldsymbol{Z}^{-1} \boldsymbol{Y}^{\mathrm{T}}=\left[T_{i l}, i=1,2, \ldots, N, l=0,1, \ldots\right.$, $M]$, then

$$
\begin{array}{r}
T_{i l}=\frac{2}{M(M+N)}\left\{(M+N) \cos \left(\frac{\left(i-\frac{1}{2}\right) l \pi}{M}\right)\right. \\
\left.-\sum_{k=1}^{N} \cos \left(\frac{l\left(k-\frac{1}{2}\right) \pi}{M}\right)\right\}
\end{array}
$$

$$
=\left\{\begin{array}{l}
\frac{2}{M+N}, \quad l=0 \\
\frac{2 \cos \left(\frac{\left(i-\frac{1}{2}\right) l \pi}{M}\right)}{M}-\frac{\sin (N l \pi) / M}{M(M+N) \sin (l \pi / 2 M)} \\
0<l<M \\
0, \quad l=M .
\end{array}\right.
$$

\section{References}

[1] M.O. Ahmad and J.D. Wang, "An analytical least-squares solution to the design problem of two-dimensional FIR filters with quadrantally symmetric or antisymmetric frequency response", IEEE Trans. Circuits Syst., Vol. CAS-36, July 1989 , pp. 968-979. 
[2] V.R. Algazi, M. Suk and C.S. Rim, "Design of almost minimax FIR filters in one and two dimensions by WLS techniques", IEEE Trans. Circuits Syst., Vol. CAS-33, June 1986 , pp. 590-596.

[3] C. Charalambous, "The performance of an algorithm for minimax design of two-dimensional linear-phase FIR digital filters", IEEE Trans. Circuits Syst., Vol. CAS-33, June 1986, pp. 590-596.

[4] J.V. Hu and L.R. Rabiner, "Design technique for twodimensional digital filters", IEEE Trans. Audio Electroacoust., Vol. AU-20, October 1972, pp. 249-257.

[5] J.H. Lodge and M.M. Fahmy, "An efficient $l_{p}$-optimization technique for the design of two-dimensional linear-phase FIR digital filters", IEEE Trans. Acoust. Speech Signal Process., Vol. ASSP-28, June 1980, pp. 308-312.
[6] J.H. McClellan, "The design of two-dimensional digital filter by transformation", Proc. 7th Ann. Princeton Conf. Inform. Sci. Syst., Princeton, USA, March 1973, pp. 247-251.

[7] S.C. Pei and J.J. Shyu, "2-D FIR eigenfilters: a least squares approach", IEEE Trans. Circuits Syst., Vol. CAS-37, January 1990, pp. 24-34.

[8] S.-C. Pei and J.-J. Shyu, "Symmetric properties of two-dimensional sequences and their applications for designing linear-phase 2-D FIR digital filters", Signal Processing, Vol. 42, No. 3, March 1995, pp. 261271.

[9] L.R. Rabiner and B. Gold, Theory and Application of Digital Signal Processing, Prentice-Hall, Englewoods Cliffs, NJ, 1975, pp. 77-84. 